\title{
əImproving Meteorological Seasonal Forecasts for Hydrological Modeling in European Winter
}

\author{
NiCKY STRINGER, JEFF KNIGHT, AND HAZEL THORNTON \\ Hadley Centre, Met Office, Exeter, United Kingdom
}

(Manuscript received 23 April 2019, in final form 18 October 2019)

\begin{abstract}
Recent advances in the skill of seasonal forecasts in the extratropics during winter mean they could offer improvements to seasonal hydrological forecasts. However, the signal-to-noise paradox, whereby the variability in the ensemble mean signal is lower than would be expected given its correlation skill, prevents their use to force hydrological models directly. We describe a postprocessing method to adjust for this problem, increasing the size of the predicted signal in the large-scale circulation. This reduces the ratio of predictable components in the North Atlantic Oscillation (NAO) from 3 to 1. We then derive a large ensemble of daily sequences of spatially gridded rainfall that are consistent with the seasonal mean NAO prediction by selecting historical observations conditioned on the adjusted NAO forecasts. Over northern and southwestern Europe, where the NAO is strongly correlated with winter mean rainfall, the variability of the predicted signal in the adjusted rainfall forecasts is consistent with the correlation skill (they have a ratio of predictable components of $\sim 1$ ) and are as skillful as the unadjusted forecasts. The adjusted forecasts show larger predicted deviations from climatology and can be used to better assess the risk of extreme seasonal mean precipitation as well as to force hydrological models.
\end{abstract}

\section{Introduction}

Seasonal hydrological forecasting offers the opportunity to prepare for events such as floods and droughts that can have a large impact on society. For example, reservoir levels can be managed to prepare for higher-than-normal precipitation to reduce the risk of flooding or increase the generation of hydropower (Hamlet et al. 2002; Anghileri et al. 2016; Viel et al. 2016). Hydrological conditions during a season can depend on both the initial state of the terrestrial water stores and the meteorological conditions that happen throughout the season. The relative influence of each factor varies from river basin to river basin ( $\mathrm{Li}$ et al. 2009; Wood and Lettenmaier 2008). One approach to hydrological forecasting that exploits the influence of the initial conditions is ensemble streamflow prediction (ESP; Day 1985). This method initializes a hydrological model with the hydrological state (soil

Denotes content that is immediately available upon publication as open access.

Corresponding author: Nicky Stringer, nicky.stringer@ metoffice.gov.uk moisture, snowpack, etc.) and is forced with an ensemble of previously observed meteorological conditions. This gives a range of possible outcomes for the season given the known initial state. However, this method relies entirely on the long-term "memory" of the hydrological system at the start of the season and gives no weighting to particular meteorological conditions during the season. This means that ESP is most skillful for river basins that are more sensitive to the initial hydrological conditions. It has been used, for example, in parts of western United States since the 1970s (Yuan et al. 2015), where the hydrological system is dependent on the snowpack accumulation over winter and then snowmelt in the spring. The lack of sensitivity to the concurrent meteorological conditions hinders the application of this method to areas that are less dependent on the initial hydrological state. If seasonal forecasts of the meteorological conditions were to be used, the skill of this method could be improved in areas that are more sensitive to the meteorology throughout the season. This would narrow the range of possible meteorological outcomes and allow the likelihood of different scenarios to be assessed. This principle was used by Beckers et al. (2016), for example, who made hydrological forecasts for several 
river basins in the United States by conditioning the selection of meteorological scenarios on the El NiñoSouthern Oscillation (ENSO) conditions at the start of the forecast period, showing an increase in the skill of the streamflow forecasts. Although this application does not use seasonal forecasting explicitly, the initial ENSO state is likely to persist throughout the season and influence the meteorological conditions.

Traditionally, skillful seasonal meteorological forecasts have been confined to the tropics. However, in recent years, there has been an emergence of skill in the prediction of large-scale circulation patterns in the midlatitudes, especially during winter. In this study we focus on European seasonal meteorological forecasts and their hydrological applications. The most important season for groundwater recharge in Europe is winter. In southern Europe, this is the season that provides the majority of rainfall in the year. In northern Europe, higher evapotranspiration levels in the summer means that the infiltration of precipitation into terrestrial water stores is reduced, and therefore groundwater recharge occurs mainly in winter. This makes the precipitation received over winter important for the hydrological situation throughout the year.

For Europe, the dominant atmospheric circulation pattern associated with the year-to-year changes in winter climate is the North Atlantic Oscillation (NAO) (Hurrell 1995). The magnitude and sign of the NAO index indicate the strength and location of the storm track. A positive NAO corresponds to enhanced westerly flow over the midlatitude North Atlantic Ocean and a more northerly storm track, whereas a negative NAO corresponds to more frequent anticyclonic blocking and a weak or diverted storm track (Hurrell et al. 2003). Since the precipitation over the winter period is mostly a result of a sequence of cyclonic systems, the state of the storm track has an impact on the spatial distribution of the winter mean precipitation.

Figure 1 shows the correlation between the observed December-February (DJF) mean NAO and precipitation between 1950 and 2016. This shows that the correlation is not uniform over Europe but is high in specific regions such as parts of the United Kingdom, Scandinavia, and the Iberian Peninsula, and that when the NAO is in a positive phase there are positive precipitation anomalies over northern Europe and negative anomalies in southern Europe, and vice versa. The NAO has also been shown to be correlated to the number and type of extreme weather events over the winter (Scaife et al. 2008).

The skill of seasonal meteorological forecast systems at predicting the winter NAO has improved greatly in recent years, with Scaife et al. (2014) reporting a correlation with
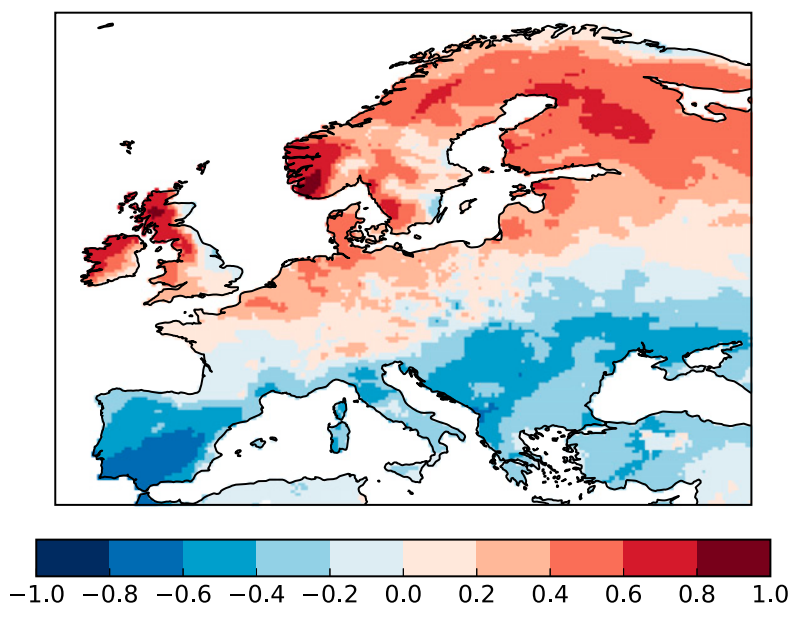

FIG. 1. Correlation of DJF mean precipitation (E-OBS; Haylock et al. 2008) with NAO between 1950 and 2016. The NAO is calculated as the seasonal mean difference between the (MSLP anomalies in the grid boxes over Gibraltar and Reykjavik (MSLP observations are from HadSLP2; Allan and Ansell 2006).

observations of 0.62 in the Met Office's Global Seasonal long-range prediction system (GloSea5), forecasts and Dunstone et al. (2016) finding that even winters a year ahead can be predicted with modest skill (correlation coefficient $r=0.42$ ). If these forecasts could be used as meteorological input to hydrological models, an improvement in the seasonal hydrological forecasts should be seen in the areas with a strong positive or negative correlation between the precipitation and NAO, particularly in locations that are less sensitive to initial conditions.

There have been previous studies exploiting the link between the NAO and the European winter climate for other sectors. For example, winter U.K. transport impacts, energy demand, wind power supply, and Baltic Sea ice cover can all be skillfully predicted using the winter NAO seasonal forecasts (Palin et al. 2016; Clark et al. 2017; Thornton et al. 2019; Karpechko et al. 2015). There have also been attempts to use seasonal forecast information to predict hydrological phenomena. Baker et al. (2018a) used large-scale pressure patterns to predict the winter mean precipitation over several regions of the United Kingdom, and Svensson et al. (2015) showed that forecasts of winter NAO can be used to derive the winter mean river flows over northwestern regions in the United Kingdom using a statistical model. These studies show that there is valuable information in the seasonal meteorological forecasts; however, the two hydrological examples only give seasonal mean quantities over spatially aggregated regions. When flooding events occur, they are often the result of a period of a few days with exceptional rainfall. For example, the 
Cumbria floods in 2015 were caused by a particular low pressure system, Storm Desmond, and although the winter mean rainfall over the United Kingdom was high, the record levels of rainfall in this region fell over a 48-h period (Met Office 2016) and caused severe flooding impacts (Marsh et al. 2016). This means that the daily sequences of rainfall within the season, and not just the seasonal mean, are also important when assessing the risk of extreme events.

Given the skill of winter seasonal prediction for Europe, it should be possible to provide probabilistic hydrological forecasts using a combination of meteorological and hydrological modeling. In an ideal scenario, we would use the meteorological variables as they are predicted by the forecast systems; however, there have been a number of recent studies that show a signal-tonoise problem common to many seasonal forecasting systems (Eade et al. 2014; Dunstone et al. 2016; Baker et al. 2018b; Scaife and Smith 2018; Siegert et al. 2016). Eade et al. (2014) and Dunstone et al. (2016) showed that, although the year-to-year fluctuations of the NAO are skillfully predicted by GloSea5, the model is underconfident, meaning that the magnitude of the ensemble mean deviations from climatology are too small given the level of prediction skill. Whenever the observations are compared against an ensemble mean of predictions, the variability of the latter is expected to be smaller. This is because the ensemble members and observations contain both the predictable "signal" and some "unpredictable" noise, and the noise is averaged out when calculating the ensemble mean (Scaife and Smith 2018). The extent to which the ensemble mean variability is smaller than the observed variability is dependent on the size of the predictable component, which is indicated by the magnitude of the correlation between the ensemble mean and the observations. Seasonal predictions in many extratropical regions show a paradox whereby the magnitude of the variability of the ensemble mean prediction is too small given the level of correlation with the observations. Siegert et al. (2016) conducted statistical modeling of the GloSea5 winter NAO forecasts and found with very high confidence that the signal-to-noise ratio (SNR) is smaller in the model than in the observations $\left[P\left(\mathrm{SNR}_{\text {obs }}>\mathrm{SNR}_{\text {model }}\right)>0.99\right.$, where $P$ is the posterior probability in a Bayesian framework]. They went on to conclude that, while there is useful information in the ensemble mean, the ensemble members are not exchangeable with the observations, meaning they should not be treated as plausible versions of reality without postprocessing. This conclusion unfortunately limits the usefulness of the forecasts to drive hydrological models without additional postprocessing. To realize the potential that the skill in seasonal forecasts offers, we need to adjust the forecasts to account for this limitation.

In this paper we present a method to increase the signal size of the predicted winter mean NAO. We then construct a large ensemble of daily sequences of European precipitation that are consistent with the adjusted seasonal mean NAO prediction. We start by describing the GloSea5 forecasting system, then in section 3 we assess the seasonal precipitation forecasts directly from the model and outline our motivation for adjusting the forecasts. Next we describe the method we have developed, and finally we assess the results of these adjustments.

\section{Seasonal forecast data}

We use the current version of GloSea5 (MacLachlan et al. 2015) (data available from Copernicus 2019), which is built around the Hadley Centre Global Environment Model, version 3 (HadGEM3). HadGEM3 combines atmospheric, ocean, land, and sea ice model components and has an atmospheric resolution of $0.83^{\circ}$ longitude by $0.55^{\circ}$ latitude and 85 quasi-horizontal atmospheric levels, with a well-resolved stratosphere. The ocean resolution is $0.25^{\circ}$ in both latitude and longitude, with 75 vertical levels. Forecasting for a season ahead is far beyond the deterministic range of weather forecasting; instead an ensemble is used to produce a probabilistic forecast. Two model forecast ensemble members are routinely initialized per day throughout the year, running for up to 6 months. A seasonal forecast is typically constructed by combining members initialized in the 3-week period prior to the release of the forecasts to form a lagged ensemble. For example, the ensemble forecast for DJF is normally issued around 20 November and is constructed from members initialized in the 21 days before, giving 42 members.

Retrospective predictions ("hindcasts") are also made over a historical period from 1993 to 2016 so that the skill of the model can be assessed by comparing the hindcasts with observations. The hindcasts are initialized on a weekly basis, and there are currently 17 hindcast members available per start date. For this study we combine the hindcast ensemble members from three initialization dates, giving us a 51-member ensemble. For example, the forecast for DJF combines the members initialized on 1,9, and 17 November. This means that the number of members and the initialization period are comparable between the forecast and hindcast ensembles, so the hindcast will give a representative picture of the skill of the forecast.

In this study we assess the 51-member hindcast ensemble predictions of daily precipitation (referred to 
as the "raw" ensemble). We also use the mean sea level pressure (MSLP) to calculate the NAO as the seasonal mean difference in the MSLP anomalies between the model grid boxes over Reykjavik (Iceland) and Gibraltar. We present the results for the DJF period; however, we have also conducted the analysis over the January-March period.

We compare the model predictions over the hindcast period to observations to assess their skill. For precipitation we use the "E-OBS" dataset (Haylock et al. 2008; ECA\&D 2019), which gives gridded daily precipitation values over Europe (land only) from 1950 to present based on station observations. For MSLP observations we use the "HadSLP2" dataset (Allan and Ansell 2006; Met Office 2019), which provides spatially gridded monthly MSLP fields from 1850 based on global station and marine observations.

\section{Motivation: Raw model skill assessment}

First we assess the skill of the unadjusted GloSea5 predictions of seasonal mean precipitation over the hindcast period. We are interested in the ability of the forecast system to predict the year-to-year deviations of the seasonal mean precipitation from climatology, and also the magnitude of the variability of the predicted signal, given by the ensemble mean. The latter is particularly important given the ultimate application of the forecasts to force hydrological models. The former is summarized by the correlation between the forecast ensemble mean and observations shown in Fig. 2.

The correlation map shows that the areas of skillful precipitation forecasts (correlation above 0.4 ) are found where there is a strong link between the winter mean precipitation and the NAO (shown previously in Fig. 1), for example, the Iberian Peninsula, parts of the United Kingdom and Ireland, and Scandinavia. This is expected given the known skill in the GloSea5 NAO forecasts (Scaife et al. 2014) and indicates that there is potentially useful information in the seasonal forecast for hydrological applications.

Next we assess the magnitude of the variability of the predicted signal. As outlined in the introduction, the variability of an ensemble mean is expected to be smaller than the observations due to the cancellation of noise between the ensemble members. The degree to which this is the case depends on the signal-to-noise ratio in the ensemble members, which in turn depends on the magnitude of the predictable signal in the observations. This is summarized by the ratio of predictable components (RPC) introduced by Eade et al. (2014) and defined as

$$
\mathrm{RPC}=r /\left(\sigma_{\text {sig }}^{2} / \sigma_{\text {tot }}^{2}\right)^{1 / 2}
$$

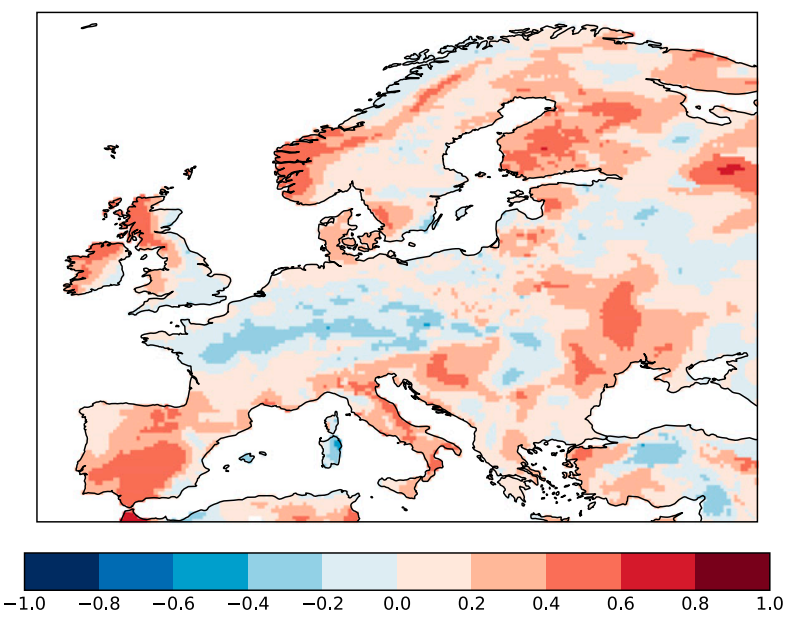

FIG. 2. Correlation of DJF ensemble mean, seasonal mean precipitation with observations (E-OBS) over the hindcast period (1993-2016) for the raw GloSea5 hindcast.

where $r$ is the correlation between the ensemble mean and observations, $\sigma_{\text {sig }}^{2}$ is approximated by the ensemble mean variance, and $\sigma_{\text {tot }}^{2}$ is approximated by the total variance across all members. An RPC value of $\sim 1$ means that the size of the predicted signal from the forecast system is consistent with the fraction of the observed variability that is predicted by the forecast system. If the RPC is much less than 1, the signal-tonoise ratio in the ensemble members is too large and the forecast system is overconfident, with the ensemble members being similar to each other but relatively dissimilar to the observations. If the RPC is much greater than 1 , the signal-to-noise ratio in the ensemble members is too small and the forecast system is underconfident, with a reasonable correlation between the ensemble mean and observations but more variability between the ensemble members. Eade et al. (2014) showed that the GloSea5 predictions of mean sea level pressure have an RCP of 2 or more over the European and North Atlantic region. This means that the ensemble mean has a smaller variability than the overall variability and the correlation would imply. The RPC is shown for the unadjusted GloSea5 hindcasts of DJF mean precipitation in Fig. 3. We have masked regions where the correlation is below 0.2. A low correlation means that the predictable variability $\left(\sigma_{\text {sig }}^{2}\right)$ is low and means that the RPC [Eq. (1)] is poorly defined. It can be seen that for most of the regions where the correlation is larger than 0.2 , the RPC is greater than 1 , showing that the variability of the ensemble mean predictions of precipitation is smaller than would be expected given the correlation skill of the model.

The signal-to-noise problem in precipitation and other variables is evidence of a deficiency in the prediction 

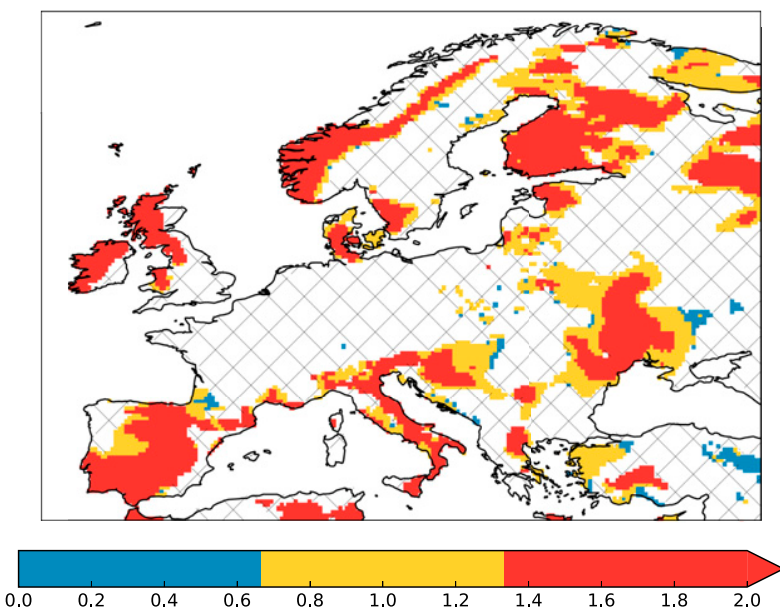

FIG. 3. Ratio of predictable components [Eq. (1)] for DJF seasonal mean precipitation predictions for the raw GloSea5 hindcast. Areas with a correlation of less than 0.2 are not shown and are hatched out.

system; however, the cause of this deficiency is not yet known. To make use of the skill in seasonal predictions of winter European climate, some postprocessing of the forecasts is required to correct for this deficiency. In the rest of this study, we present a method to enhance the size of the signal in the predictions of atmospheric circulation to a level that is consistent with the correlation skill we see in the forecasts. From there we derive the precipitation forecast that is consistent with the predicted seasonal mean circulation pattern by using historical observations. The observations are used as analogs for the predicted rainfall in place of the model. These are selected based on when the historically observed NAO is similar to that of the adjusted model NAO prediction. Our method is described in more detail below.

\section{Method to enhance the forecast signal}

Our aim is to provide seasonal forecasts of precipitation suitable for input into hydrological models in order to improve decision-making and enable better preparation for extreme events. The hydrological models require daily resolution and spatially gridded forecasts. We have shown that the signal-to-noise problem affects the winter precipitation forecasts, and we know that the driving NAO forecasts from the model are also affected, with both having an RPC of more than 2 (Scaife and Smith 2018). This means we need to adjust the forecasts to take this into account before the precipitation forecasts can be used. Given the similarity between the spatial pattern in the correlation between winter mean precipitation and the NAO shown in Fig. 1, and the correlation skill of the precipitation forecast shown in Fig. 2, we hypothesize that the skill in the precipitation forecasts originates from the skill predicting the large-scale circulation pattern indicated by the NAO. We therefore take the approach of increasing the size of the predicted signal in the NAO forecast. An ensemble of daily and spatially gridded precipitation forecasts is then constructed by selecting many historical precipitation analogs in a similar approach to the ESP method; however, in our method we condition our sampling based on the NAO seasonal forecasts. There are three key steps to achieving this:

1) increase the magnitude of the ensemble mean variability (increase the signal),

2) adjust the ensemble members to conserve the overall variability of the ensemble (decrease the noise), and

3) select historical analogs according to the adjusted NAO ensemble.

Each step is described in more detail below.

\section{a. Increasing the ensemble mean $N A O$ variability}

The time series of the observed and predicted DJF NAO (shown in Fig. 4) shows that the ensemble mean correlates well with the observations, with a correlation of 0.67 . It also shows that the variability of the ensemble mean is small when compared with the observations. To increase the variability of the predicted NAO signal, we fit a linear regression of the observed seasonal mean NAO against the predicted seasonal ensemble mean NAO. This is done using the ordinary least squares method over the hindcast period between 1993 and 2016. The slope of this regression becomes the scaling factor $\alpha$ to adjust the ensemble mean NAO. For DJF, $\alpha=2.7$ (ranging between 2.2 and 3.2 when we perform a leave-one-out cross validation). The magnitude of the slope parameter is comparable to the RPC of the NAO, which is 3.11, so this scaling factor is expected to reduce the RPC to around 1.

\section{b. Adjusting the ensemble members to conserve their total variability}

Since the standard deviation of the DJF mean NAO in individual members $(6.5 \mathrm{hPa})$ is comparable to that of the observations $(7 \mathrm{hPa})$, we would like to conserve the overall variability of ensemble members across all years. To do this we reduce the variability of the ensemble members in a particular year so that we satisfy

$$
\sigma_{\text {tot }}^{2}=\sigma_{\text {mean }}^{2}+\sigma_{\text {members }}^{2}=\sigma_{\text {mean }}^{\prime 2}+\sigma_{\text {members }}^{\prime 2},
$$

where $\sigma_{\text {mean }}^{2}$ is the variance of the ensemble mean and $\sigma_{\text {members }}^{2}$ is the variance of the ensemble members around the mean, and the primed quantities are the adjusted variances. Given that $\sigma_{\text {mean }}^{\prime 2}=\alpha^{2} \sigma_{\text {mean }}^{2}$, we can rearrange Eq. (2) to give the adjusted ensemble member variance as 
(a) DJF NAO

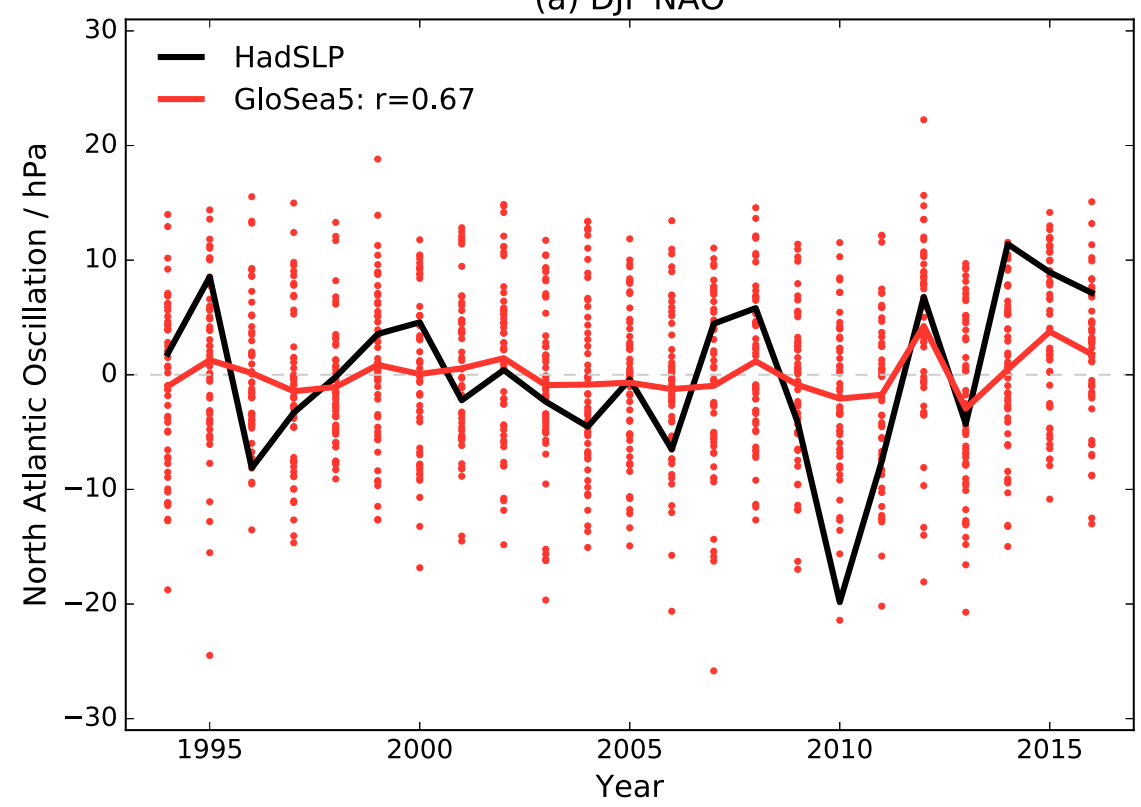

(b) DJF Rescaled NAO $(\alpha=2.7)$

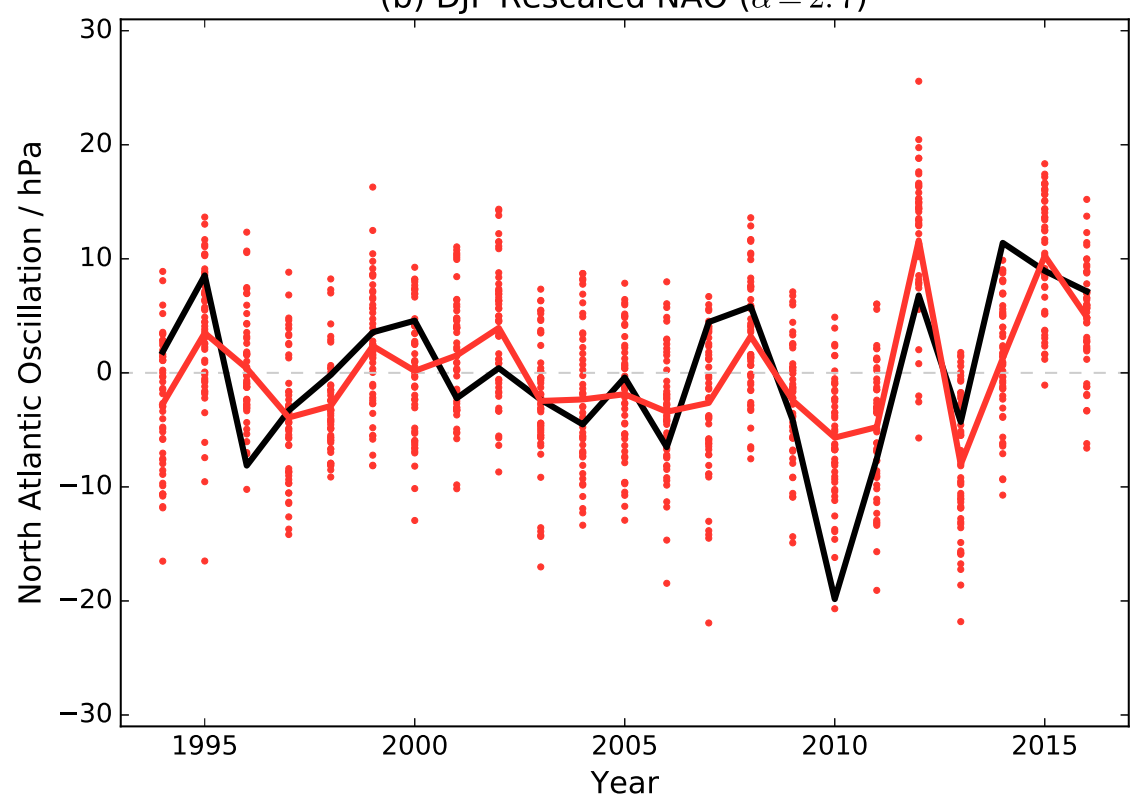

FIG. 4. Time series of the DJF mean NAO from the (a) raw and (b) adjusted GloSea5 ensemble mean (red lines) over the hindcast period (1993-2016). The ensemble members are shown by the red dots, and the black line shows observations from HadSLP2 (Allan and Ansell 2006) in both cases. The year on the $x$ axis refers to the year of the January and February of the DJF period. So 2010 refers to DJF 2009/10.

$$
\sigma_{\text {members }}^{\prime 2}=\left(1-\alpha^{2}\right) \sigma_{\text {mean }}^{2}+\sigma_{\text {members }}^{2}
$$

This is the same as the method used in Eade et al. (2014).

The impact of rescaling the NAO is shown in Fig. 4b where greater year-to-year variability of the ensemble mean $\mathrm{NAO}$ is seen, going from $\sigma_{\text {mean }}=1.4 \mathrm{hPa}$ in the raw hindcast to $\sigma_{\text {mean }}^{\prime}=4.4 \mathrm{hPa}$ in the adjusted hindcast.
The linear scaling of the ensemble mean NAO means the correlation remains unchanged at 0.67 . It can also be seen that the spread of the ensemble members around the mean has been reduced, which conserves the total variability across all the members with $\sigma_{\text {tot }}=6.5 \mathrm{hPa}$. The variability of the adjusted ensemble mean is still smaller than the observations, as it should be given the 
correlation skill is less than unity nevertheless, our adjustments have increased the size of the signal to a level where it is now consistent with the skill of the forecasts. If we approximate $\sigma_{\text {sig }}^{2}$ as $\sigma_{\text {mean }}^{2}$ and then substitute into Eq. (1) the values of $\sigma_{\text {sig }}, \sigma_{\text {tot }}$, and $r$, we can see that the RPC has reduced from 3.11 to 0.99 , meaning that our adjustments to the NAO have been successful.

\section{c. Selection of analog months}

Following the adjustment of the seasonal mean NAO to increase the size of the predicted signal, we need to construct daily realizations of precipitation that are consistent with this adjusted seasonal mean NAO. These fields can then be used as input to hydrological models. We do this by selecting historical precipitation analogs using the adjusted NAO as a constraint. The difference between the NAO predicted by the model ensemble member and that in the HadSLP observations is calculated. All observed dates that have a difference that is smaller than a threshold (defined in the next section) are available to be randomly selected. We then take the sequence of observed daily mean precipitation from the selected date as our forecast. This is a similar approach to ESP (Beckers et al. 2016) but is conditioned on the predicted NAO. The advantages of selecting from the observations is that model biases are avoided, and any observations dataset can be used provided that it spans the sampling period, which allows the hydrological modeler to sample from their own datasets. Using historically observed analogs based on the atmospheric circulation pattern to represent surface meteorological variables has been used in climate studies before. Zorita and Von Storch (1999) described an analog method to downscale lower-resolution climate simulations to local meteorological variables for use in impacts studies. Yiou et al. (2007) used analogs to show that the anomalously warm autumn-winter 2006/07 period was inconsistent with having been caused entirely by circulation patterns over the season. In this study we use the $\mathrm{NAO}$ as our metric to select the analogs to represent the seasonal forecasts.

\section{1) Analog dataset}

We have chosen to use observations as our analog dataset, and use the E-OBS dataset for this study (Haylock et al. 2008), which gives daily gridded rainfall over Europe at $0.25^{\circ}$ resolution from 1950 to the present day, based on station observations. The E-OBS dataset covers the period between 1950 and 2017, giving 67 DJF seasons from which to sample. Thompson et al. (2017) have shown that because the observed record is limited in duration, the likelihood of extreme seasons may be underestimated by using historical conditions to define the range of possible outcomes. Here we investigate the limitations of the relatively small analog sample size and if this can be mitigated. Figure 5 shows the distribution of the winter mean NAO in HadSLP2 observations and in the adjusted GloSea5 ensemble members over the hindcast period. The winter of 2009/10 (plotted as the outlier) stands out as having an exceptionally negative $\mathrm{NAO}$ in the observations. It is more than 3 standard deviations below the whole HadSLP 1850-2017 mean, and the lowest in the whole record. Prior to this season, inspection of the observed record would have given no indication that this seasonal mean value of the NAO was possible. As well as being the minimum observed value, it is $6 \mathrm{hPa}$ below the next most negative NAO, highlighting how much of an outlier that DJF period was. This winter is available to sample as a historical analog; however, we have chosen to treat it as an outlier and exclude it from the next piece of analysis to give a more representative picture of the observed NAO distribution. The median and interquartile range are similar between the observed and adjusted GloSea5 distributions; however, the range (not including 2009/10) is smaller in the observations. The red crosses show events that occur in the model ensemble that are unprecedented in the observations. There are 105 DJF seasons in the hindcast that are beyond the range of what has been observed (not including 2009/10). The differences in the distributions could be caused by the limited sample size of the observations (67) relative to the model $(23$ years $\times 51$ realizations $=1173)$ or the different sampling periods (1950-2017 in the observations and 1993-2016 in the model). To investigate this further, we repeatedly sampled the adjusted GloSea5 ensemble 67 times and found a distribution of estimates for the maximum and minimum values, as well as the range. These distributions were not inconsistent with the observations (not shown), leading us to conclude that the sample size is at least part of the reason for the difference between the distributions. If we were to select our seasonal NAO value directly from the observations, we would not be able to reproduce the range of values that the prediction system suggests is possible, and we would likely underestimate the chance of extreme seasonal NAO values. Also, any predicted season with an NAO of less than $-10 \mathrm{hPa}$ would sample exclusively from DJF 2009/10. To try to avoid this problem we allow the selection of individual months from different winters in the sampling of historical analog data. We use the predicted monthly NAO values to select the analog so that each season is constructed with months from different years, for example: December 1978, January 2014, and February 1955. This means we are increasing the number of different seasons we can construct (from $N=67$ 


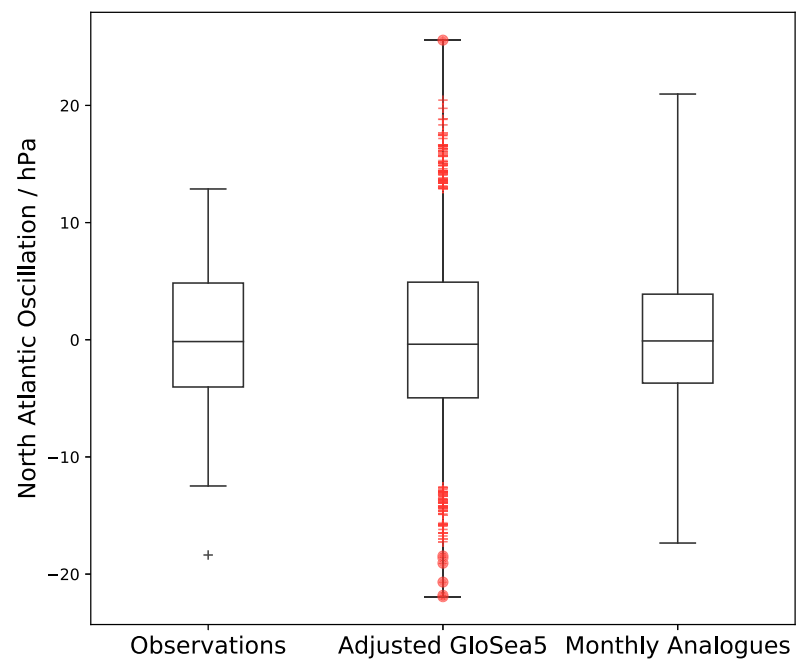

FIG. 5. Boxplots showing the distribution of DJF-mean NAO from (left) HadSLP observations between 1950 and 2017, (center) the adjusted 51-member GloSea5 ensemble over the hindcast period between 1993 and 2016, and the NAO resampled from monthly observed analogs. The red plus signs indicate events in the GloSea5 ensemble that fall beyond the range of the observations (not including 2009/10), and filled red circles indicate events that fall beyond the range of the resampled monthly analogs. The 2009/10 winter is treated as an outlier and is not included in the range of the observations. This point is shown by the black plus sign in the left boxplot.

to $N=67^{3}=300763$ ), and we are able to construct seasons with previously unobserved NAO values. Figure 5 shows the distribution of the seasonal mean NAO sampled from observations in this way. We can see that the number of extreme seasons that are outside the range of the adjusted GloSea5 predicted seasonal NAO is much reduced, going from 105 in the observations to 8 in our resampled hindcast. This means that we are able to reproduce the range of predicted NAO values with the observations when constructed from individual months. A consequence of sampling the months separately is that there will be a discontinuity in the daily sequence at the transition between months; however, it is anticipated that the impact of this will be small since the autocorrelation in European winter daily precipitation is relatively low. We conclude that it is appropriate to use the observations dataset to sample the analogs from, when the season is split into months.

\section{2) Selecting multiple analogs}

An important consideration in selecting analogs is that although the NAO is a strong predictor of winter precipitation, it does not explain all of the variability. There is also a component that is not influenced by the NAO. As our predictor is the set of NAO values from the ensemble prediction system, we wish the analog outcome to reflect the NAO phases in the ensemble rather than any idiosyncratic features of the precipitation that was present in a selected month. We do this by selecting multiple analogs for a single NAO prediction. As these analogs all have similar NAO values, the NAO influence across this set of realizations is consistent, whereas the impact of any non-NAO-related variability in the set as a whole will be reduced as more analogs are added. To sample several analogs, we select the samples from a selection window centered on the monthly NAO prediction. Any NAO observations lying within that window are candidate analogs. We then select the analogs randomly several times with replacement (since reselection of a particular month is highly unlikely to lead to a replicated 3-month season) until we have several realizations for each of our predicted NAO ensemble members.

We must choose both the appropriate size for the sampling window, and the appropriate number of analogs to select in order to minimize the influence of nonNAO-related variability. If the sampling window size is too small, the number of observed months to choose from will be too low. This means that the candidate analog months will be resampled more frequently, reducing the effective sample size and therefore not reducing the influence of non-NAO-related variability. However, if the sampling window size is too large, the range of NAO values across the collection of analogs will be too diverse and the NAO signal across the samples will be inconsistent.

First we assess the number of analogs we need to sample. Figure 6 shows how the robustness of the skill of the predicted seasonal mean precipitation varies according to the number of analogs per NAO forecast. We would like the skill of the forecasts to be robust such that the results would not change significantly if repeated. This means we want the spread of the results (indicated by the shading in Fig. 6) to be as narrow as possible. We see that the results are less robust for lower resample numbers, with large differences in the overall skill when the analog sampling is repeated. As the number of resamples increases, the spread of the results decreases, and it remains approximately constant after about six resamples. We conclude that the more resamples that can be used the more robust the predictions will be, although this tendency has limitations because of the finite size of the 51-member GloSea5 ensemble. In practice, users may have data-handling limitations. In this case, we recommend using at least six resamples per forecast system ensemble member.

Next we consider the size of the sampling window around the NAO prediction. As discussed above, there 


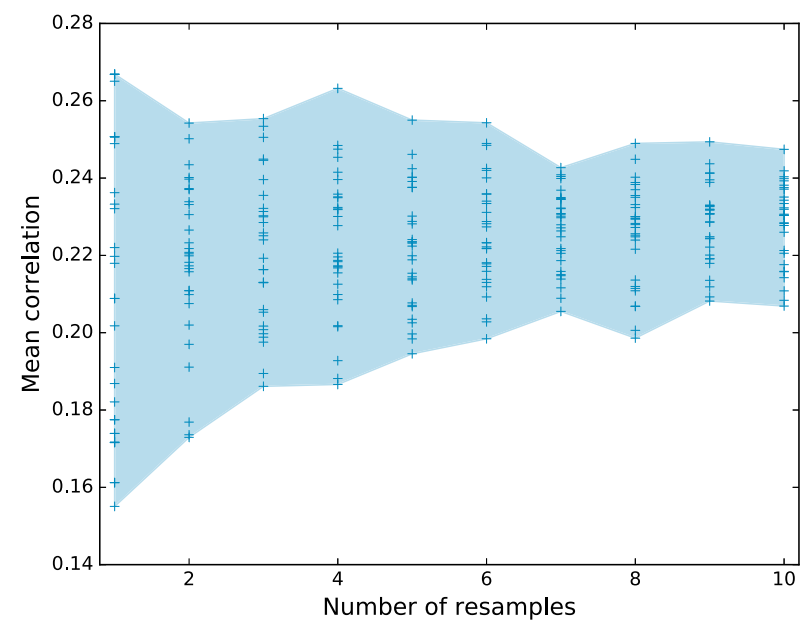

FIG. 6. Mean correlation between predicted and observed DJFmean precipitation taken over the points at which the absolute correlation between the NAO and precipitation shown in Fig. 1 is above 0.35 . This is plotted against the number of times the analog for each ensemble member is resampled. For this experiment, the analogs were sampled 20 times, and the blue plus signs show the results from the subselection of 20 trials. A selection window of $4 \mathrm{hPa}$ around the ensemble member NAO prediction is used.

is a compromise between having a large sampling pool around the NAO prediction, and the representativeness of the analog NAO values. This compromise is illustrated by Fig. 7. We test three sampling window sizes: 2 , 4 , and $6 \mathrm{hPa}$ (these sizes referring to the half-width of the window). The histograms in the top three panels show how the distribution of the number of independent samples around each GloSea5 forecast member changes as the sampling window increases. It was seen in Fig. 6 that, the larger the number of independent samples is, the more robust the precipitation forecast will be as a result of the averaging out of non-NAO-related variability. These histograms show that the number of independent samples of the historical analogs is sensitive to the sampling window. A window of $2 \mathrm{hPa}$ leads to $46 \%$ of the GloSea5 ensemble members being resampled with fewer than six independent analogs. This more than halves to $20 \%$ for a sampling window of $4 \mathrm{hPa}$ and nearly halves again with a $6-\mathrm{hPa}$ window of $11 \%$. This means that as the sampling window increases so does the number of different analog months that can be sampled.

Despite the increase in the number of available selection candidates, there is a cost in having a larger sampling window size. The scatterplots in the lower three panels of Fig. 7 show how well the predicted NAO is represented by the 10 resampled analog NAO values. We would like the difference between these values to be as small as possible. There appears to be two distinct behaviors. Moderate NAO values are clustered around the zero line, and more extreme NAO values in the tails of the distribution are much more scattered. First we focus on moderate NAO values. For a $2 \mathrm{hPa}$ sampling window, the difference between the predicted and resampled NAOs is small with no systematic bias. However, as the sampling window increases, a systematic bias is introduced, with a positive bias for negative NAO values and vice versa. This happens because the distribution of NAO values within the sampling window is not uniform. There are more samples to choose from in the half of the sampling window nearer to zero because of the overall distribution of NAO values. This bias gets larger as the sampling window size increases.

Now we focus on the more extreme values of the $\mathrm{NAO}$, for which the difference between the predicted and resampled NAOs is much larger. When the predicted NAO is in the tails of the observed distribution or beyond, the number of analogs within the sampling window can be as low as one or even zero. When this is the case, we can either chose to ignore these ensemble members or expand the window. The first option would systematically underrepresent the tails of the forecast ensemble, so we have chosen the latter. Where there are fewer than two possible candidates to select, we gradually increase the window size by $50 \%$ until there are at least two analogs to sample. This means that the difference in the resampled and predicted NAO can be larger than the original size of the sampling window. This can be seen in the large scatter of points in the tails of the NAO distribution in Fig. 7 with all sampling window sizes.

The size of the sampling window is therefore a compromise between having a large enough sampling pool and having an accurate representation of the predicted NAO. For this study we have decided to use a sampling window size of $4 \mathrm{hPa}$ (center plots in Fig. 7). This means approximately $80 \%$ of the NAO values in the hindcast period are resampled with a pool of at least six independent analogs, and the variability of the ensemble mean NAO forecast is still well represented.

\section{Results: Adjusted model skill assessment}

We have now established the method for generating our winter seasonal forecasts of daily European precipitation using the NAO. Our aim has been to increase the size of the signal to account for the signal-to-noise problem present in the raw NAO and precipitation forecasts. In doing this, we also expect to maintain the precipitation forecast skill as shown by the correlation with observations. We can test whether our method has achieved this by comparing the raw and adjusted RPC 
(a) Sampling Threshold: $2 \mathrm{hPa}$

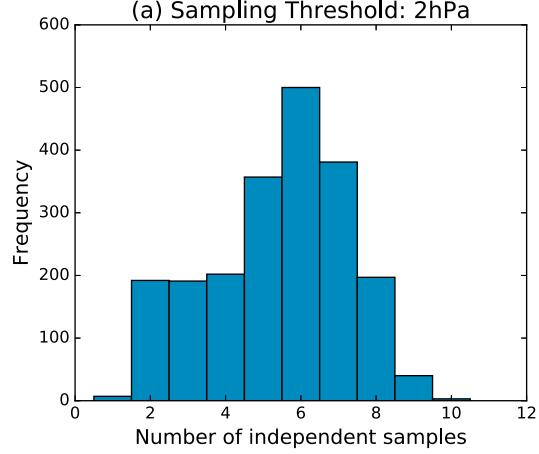

(d) Sampling threshold: $2 \mathrm{hPa}$

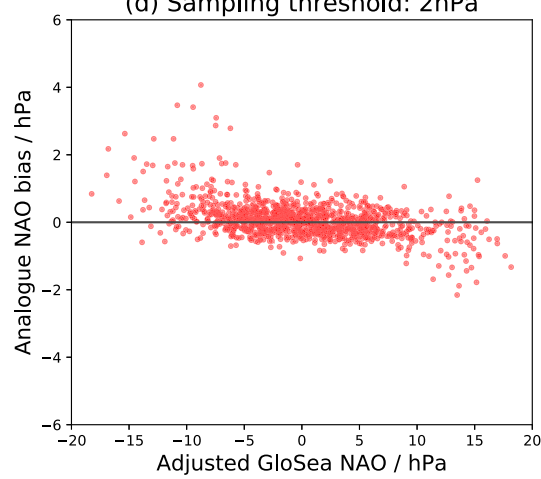

(b) Sampling Threshold: $4 \mathrm{hPa}$

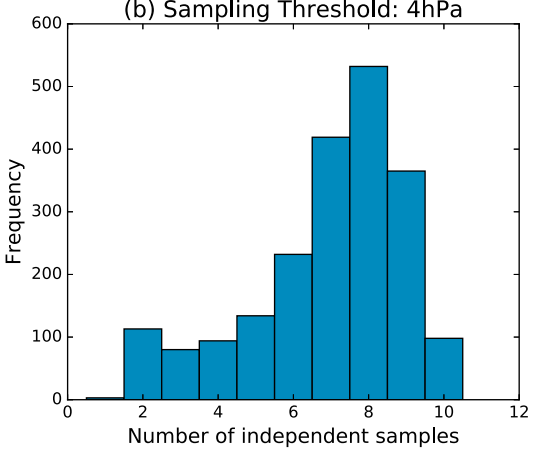

(e) Sampling threshold: $4 \mathrm{hPa}$

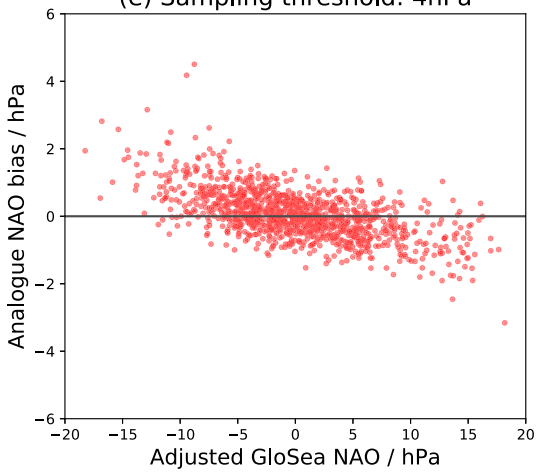

(c) Sampling Threshold: $6 \mathrm{hPa}$

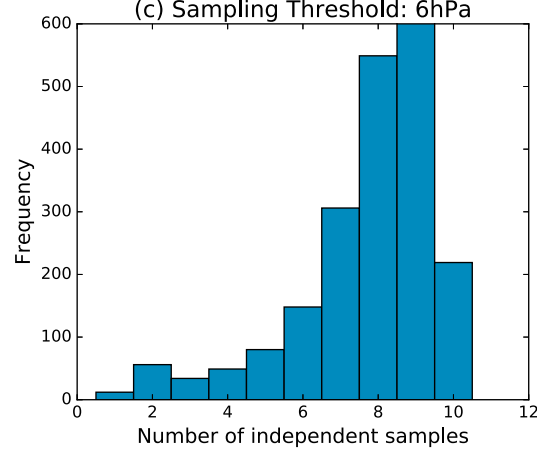

(f) Sampling threshold: $6 \mathrm{hPa}$

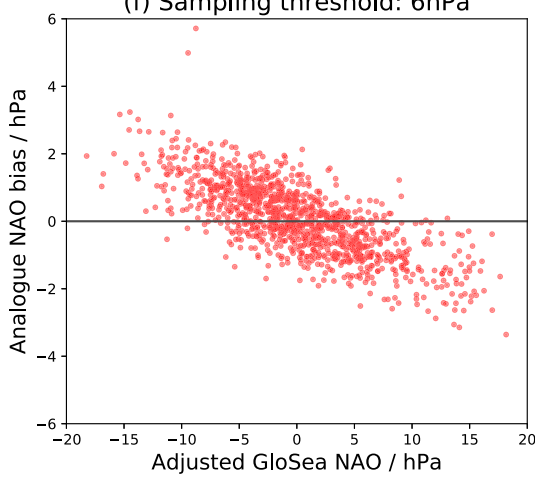

FIG. 7. (a)-(c) Histograms showing the distribution of the number of independent samples for each GloSea5 forecast ensemble member for different sampling window sizes. (d)-(f) Scatterplots showing the bias of the resampled analog NAO against the adjusted GloSea5 NAO amplitude. The NAO predicted by each adjusted GloSea5 ensemble member is resampled 10 times. The red dots show the difference between the mean NAO of the 10 resamples and the adjusted GloSea5 ensemble member against the predicted adjusted GloSea5 ensemble member NAO.

of the winter mean precipitation (Fig. 8) and comparing the correlation of the raw and adjusted winter ensemble mean precipitation forecasts with observed precipitation (Fig. 9). Figures 2 and 3 have been reproduced here for ease of comparison.

These figures show that the method has been successful in adjusting the precipitation forecasts to account for the signal-to-noise problem over certain regions. Over the western United Kingdom, Ireland, Norway, and Spain, the RPC has been reduced to around 1 (Fig. 8b). These areas correspond to those with a particularly strong link between winter precipitation and the NAO (shown in Fig. 1), which are the areas where we expect the method to perform well. Given our hypothesis that the skill in the raw precipitation forecasts is related to the skill in the NAO forecasts, we do not expect the correlation in the precipitation to change following our adjustments. Figure 9 shows that this is the case with similar correlation values in the two hindcasts. The difference between the correlations is found to be not statistically significantly different from zero.

While there are still a number of areas with an RPC clearly greater than 1 , these are not the regions that show correlation skill in the raw model analysis, or a very strong correlation between the NAO and precipitation in the longer-term observations (Fig. 1). These are regions where the correlation with observations has increased in the adjusted hindcast set (Fig. 9), such as northern France. This result was not expected. The length of the hindcast is 23 years, and therefore the number of points making up these correlations, which then go into the RPC calculation, is limited. It is very possible that the high correlations seen are a result of noise. This would be consistent with the RPC values we see in Fig. 8 b being high even after our adjustment. This highlights the importance of always assessing the physical sources of skill in seasonal predictions to add confidence to skill assessments, which are often performed over relatively short hindcast periods and small ensembles because of resource limitations.

So far we have shown an improvement in the size of the predicted signal, and also that the correlation of the ensemble mean rainfall remains consistent with the raw model predictions. This is only an assessment of the ensemble mean skill, which, although important because 
(a)
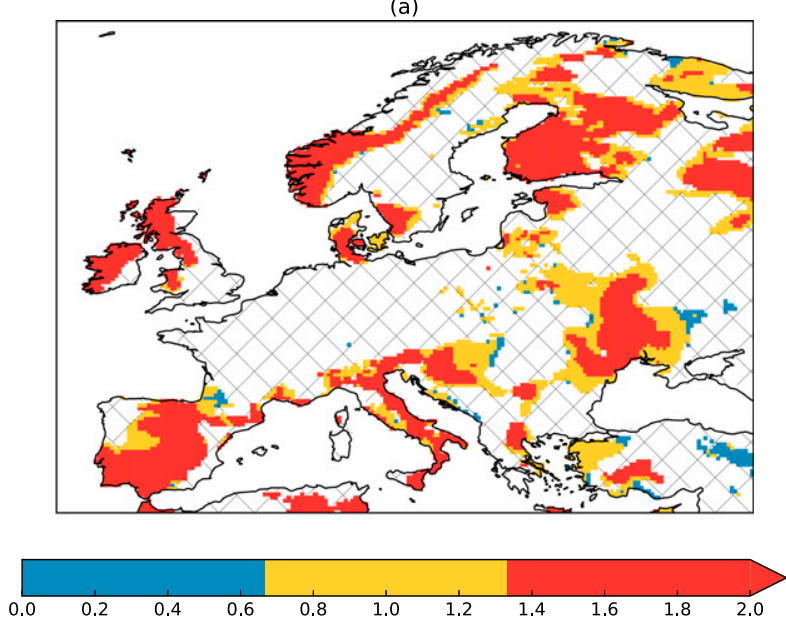

(b)
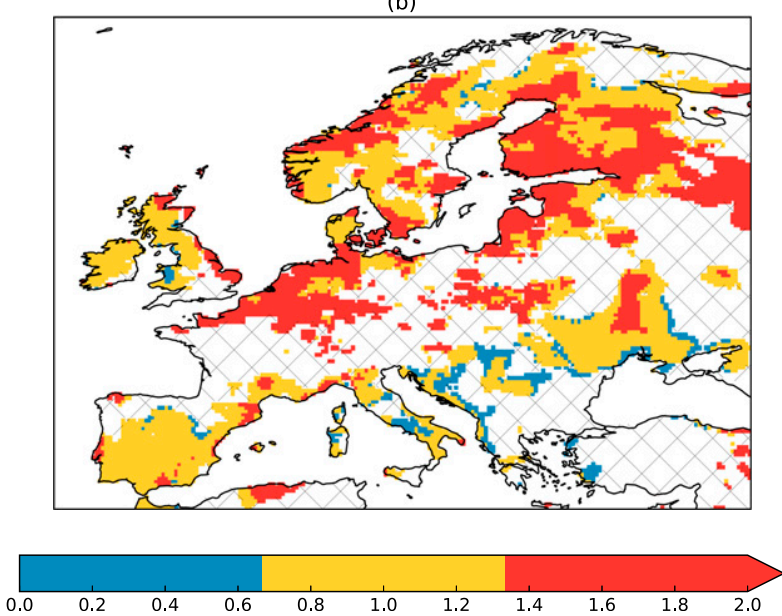

FIG. 8. Ratio of predictable components [Eq. (1)] for DJF seasonal mean precipitation predictions for (a) the raw GloSea5 hindcast (reproduced from Fig. 3) and (b) our adjusted hindcast (produced with the method discussed in section 4). Areas with a correlation of less than 0.2 are not shown and are hatched out.

it shows the predicted signal, is not the whole story. We would also like to assess the behavior of the whole model ensemble and its skill at predicting the risk of more extreme events. We do this by assessing two case study years. Figures 10 and 11 show forecasts from two example years, over two regions with different responses to the NAO. The winter of $2010 / 11$ had a negative NAO and 2015/16 had a positive NAO. Both winters had NAO magnitudes of approximately $7 \mathrm{hPa}$, which is approximately 1 standard deviation away from the mean. The NAO was well predicted by GloSea5 in both of these DJF periods, with adjusted ensemble mean predictions of $-4.8 \mathrm{hPa}$ in $2010 / 11$ and $4.8 \mathrm{hPa}$ in $2015 / 6$. These example forecasts should therefore provide a good showcase of the forecast adjustments. From the correlation and RPC maps shown
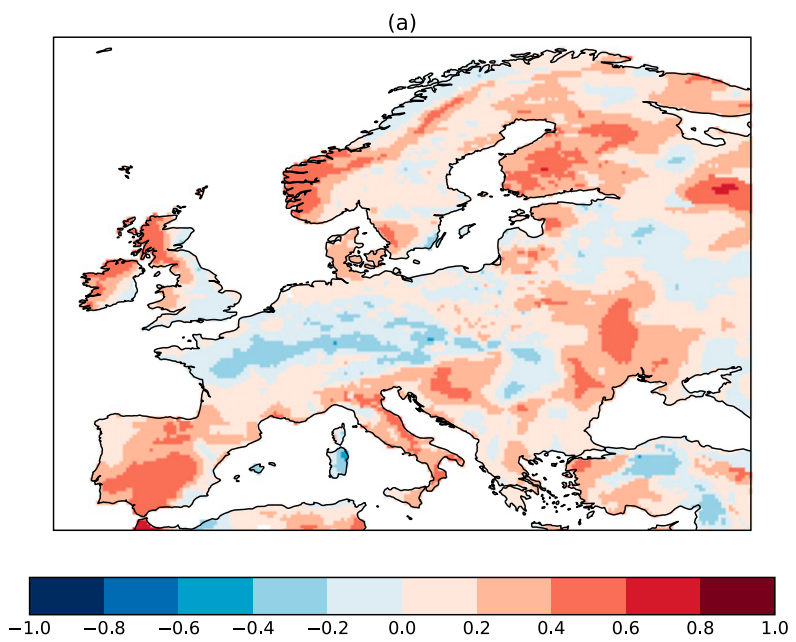

(b)
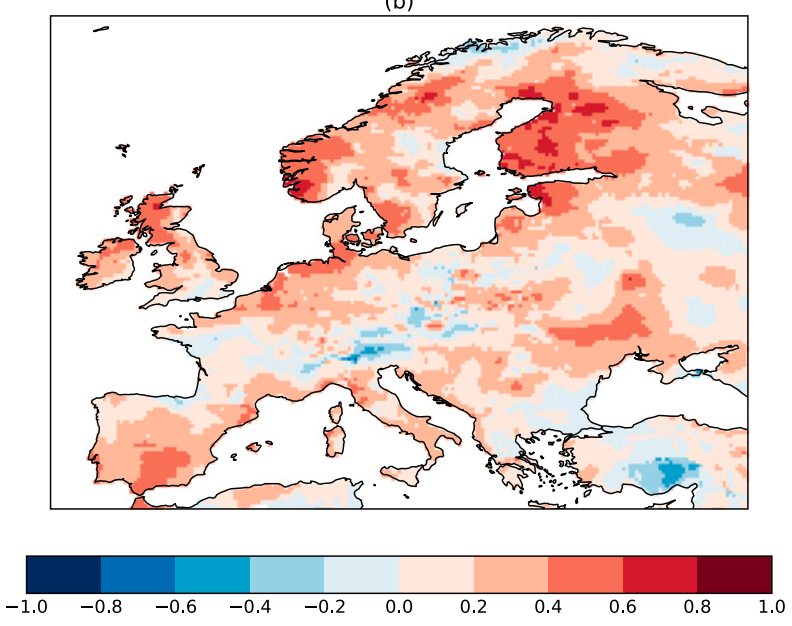

FIG. 9. Correlation of DJF ensemble mean, seasonal mean precipitation with observations (E-OBS) over the hindcast period (1993-2016) for (a) the raw GloSea5 hindcast (reproduced from Fig. 2) and (b) our adjusted hindcast (method discussed in section 4).

previously (Figs. 8 and 9) we can see that adjusted ensemble provides a skillful forecast over northern and southern (rather than central) Europe. Here we show the forecasts over small regions in the northwestern United Kingdom, which has a positive correlation between winter NAO and precipitation, and southeastern Spain, which has a negative correlation between winter NAO and precipitation. Figures 10 and 11 show the probability density functions (PDFs) of the forecast ensembles and the observed climatology. To avoid issues of undersampling, we have resampled the raw NAO forecasts and observations from the analog time series using the predicted and observed NAOs, respectively. This means that the ensemble sizes are similar allowing for a fairer and more robust comparison. The overall message is 
(a) Southeast Spain

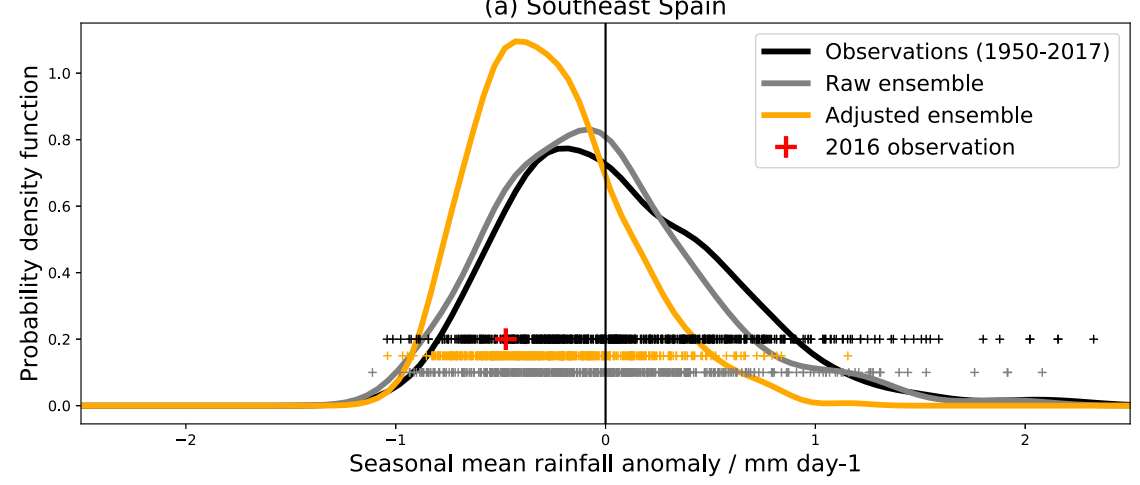

(b) Northwest UK

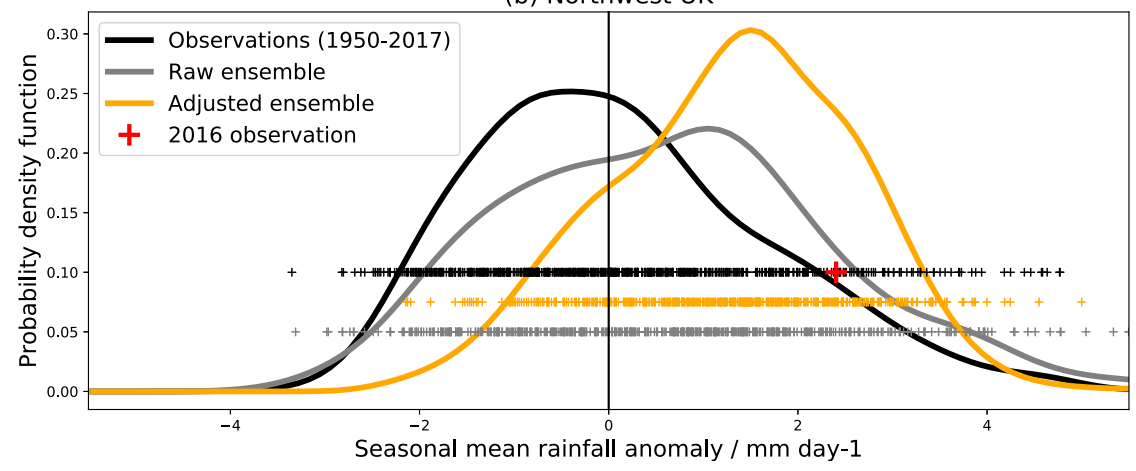

FIG. 10. DJF 2015/16 seasonal mean precipitation PDFs over (a) southeastern Spain and (b) the northwestern United Kingdom for the raw (gray) and adjusted (orange) forecast ensembles and the resampled observations (black). The adjusted and raw ensembles along with the observations have all been resampled using the method described in section 4 so that they have comparable ensemble sizes. The gray and orange plus signs show the ensemble members for the forecasts using the raw and adjusted NAO predictions, respectively. The black plus signs show the resampled precipitation using the observed NAO between 1950 and 2017. The larger red plus sign shows the observation for DJF 2015/16 from E-OBS.

consistent when looking at the raw precipitation forecasts and observations.

The winter of 2015/16 (Fig. 10) had a positive NAO and was characterized by well-above-average precipitation in the United Kingdom, particularly in the north and west. There were record-breaking periods, most notably in early December in the northwestern United Kingdom (McCarthy et al. 2016). The NAO was well predicted by GloSea5 with drivers of predictability such as ENSO and the quasi-biennial oscillation (Scaife et al. 2017).

The forecast for the northwestern United Kingdom shows a bigger shift in both the raw and adjusted rainfall distributions when compared with climatology; however, the signal is much larger in the adjusted forecast. The proportion of ensemble members above the median is $65 \%$ in the raw forecast and $86 \%$ in the adjusted forecast, with the observed rainfall lying well above the median value and in the wettest 10 seasons in our climatological period (1950-2017).

Over southeastern Spain, the observed precipitation was drier than average. The raw ensemble over southeastern Spain shows a very small deviation from the climatological distribution, with the proportion of ensemble members below the hindcast median being $63 \%$. The adjusted hindcast highlights a reduced risk of a very wet season and an increased likelihood of below-average rainfall. In this case, the proportion of ensemble members below the median increases to $82 \%$ in the adjusted forecast, showing a larger shift away from climatology. It is impossible to verify a probabilistic forecast over a single event; however, it is reassuring that the observed value lies near the middle of the adjusted distribution.

The forecast for 2010/11 (Fig. 11) had a negative NAO over DJF. Over southeastern Spain, the raw ensemble shows a small shift away from a drier-than-average season to a wetter-than-average season. $63 \%$ of the ensemble members are above the climatological median. However, again, this shift is much greater in the adjusted forecast, with $89 \%$ of the adjusted ensemble above the median. The observations are wetter than the climatological mean; however, it lies within the range of 
(a) Southeast Spain

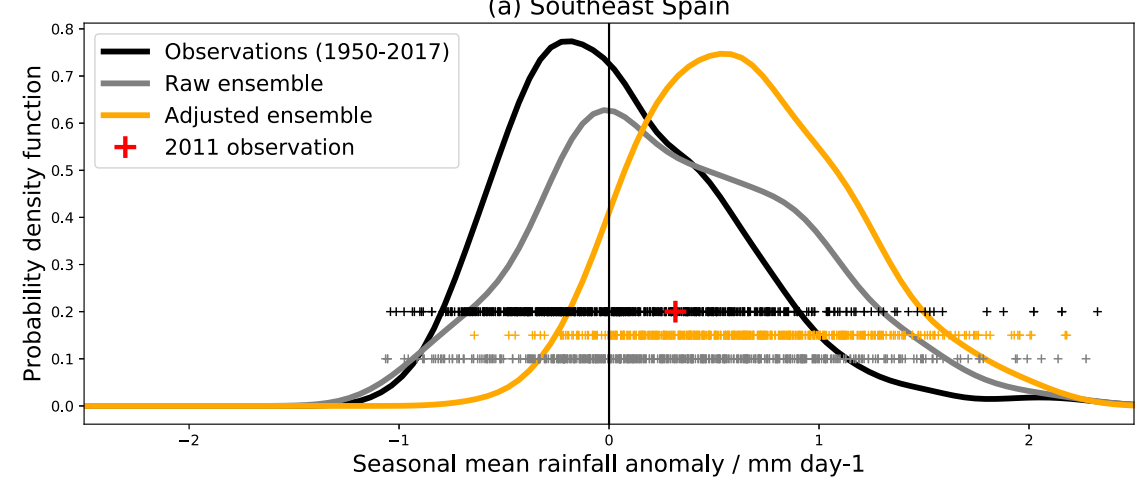

(b) Northwest UK

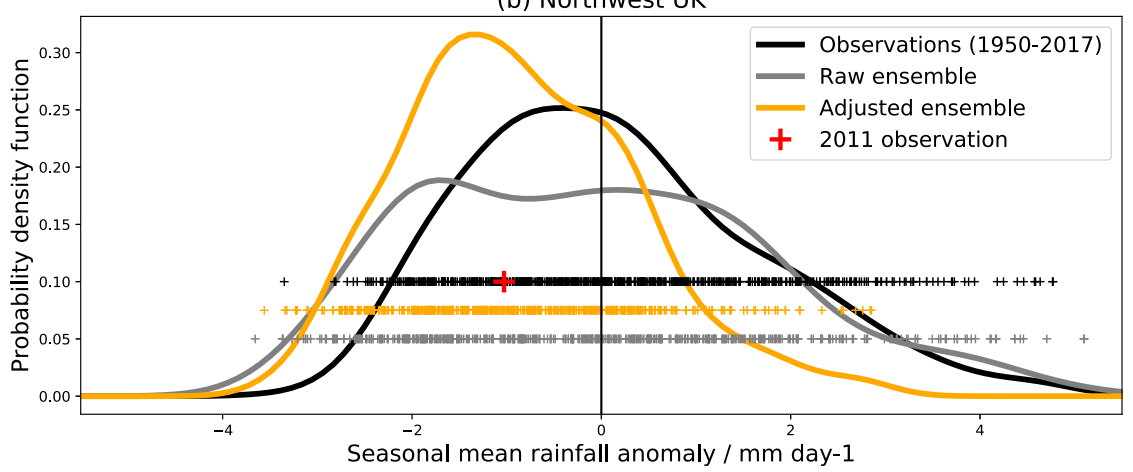

FIG. 11. As Fig. 10, but for DJF 2010/11.

both ensembles. Again, it is difficult to assess which is the better forecast from a single event, but the adjusted forecast shows a greater deviation from the climatology. Given we know that the raw ensemble mean is too close to the climatology (high RPC), we see this as an improvement in the forecast.

Over the northwestern United Kingdom, the raw forecast ensemble shows a slightly increased risk of a dry season, with a shift away from near-normal conditions rather than from wet conditions. This increases the spread of the forecast with the ensemble covering a broader range of outcomes than the climatology. This means that in fact $50 \%$ of the raw ensemble is below the median showing no strong overall signal in the forecast. In contrast, $71 \%$ of the adjusted ensemble members are below the median, and there is an indication that wetterthan-average conditions are less likely.

The results over these two seasons are promising, with larger deviations from the climatological distribution and potentially more useful forecasts. The common features across these two years and two locations are that the adjusted distributions of ensemble members are narrowed and shifted away from zero when compared against the climatological and raw distributions. These examples illustrate the overall improvement shown in the RPC and correlation maps over the whole hindcast period in Figs. 8b and 9b. To further investigate the behavior of the forecast ensembles over the whole hindcast period, Fig. 12 shows the extent to which the predicted likelihood of the seasonal mean precipitation being in the lower and upper terciles of the observed climatological distribution is increased (a "hazard increase") over the northwest of the United Kingdom. The hazard increase is calculated as the percentage increase in the likelihood of the seasonal mean precipitation being in the upper or lower tercile (whichever is more likely) relative to climatology. So, for example, a hazard increase of $-100 \%$ means there is a $100 \%$ increase (doubling) in the probability of being in the lower tercile relative to climatology. In the case of terciles, the climatological chance is $33 \%$; therefore, in this example the predicted chance is $66 \%$.

We can see in Fig. 12 that the adjusted ensemble tends to increase the magnitude of the hazard increase relative to the raw ensemble. This is because we are amplifying the size of the predicted signal, which increases the deviations away from climatology. For years when the NAO is well predicted and there is a large NAO signal (see Fig. 4b-1995, 1997, 2006, 2008, 2009, 2011, 2012, 2013, 2015, and 2016), we can see that the hazard increase is amplified in the adjusted ensemble and that the observations occur in the direction of the increased 


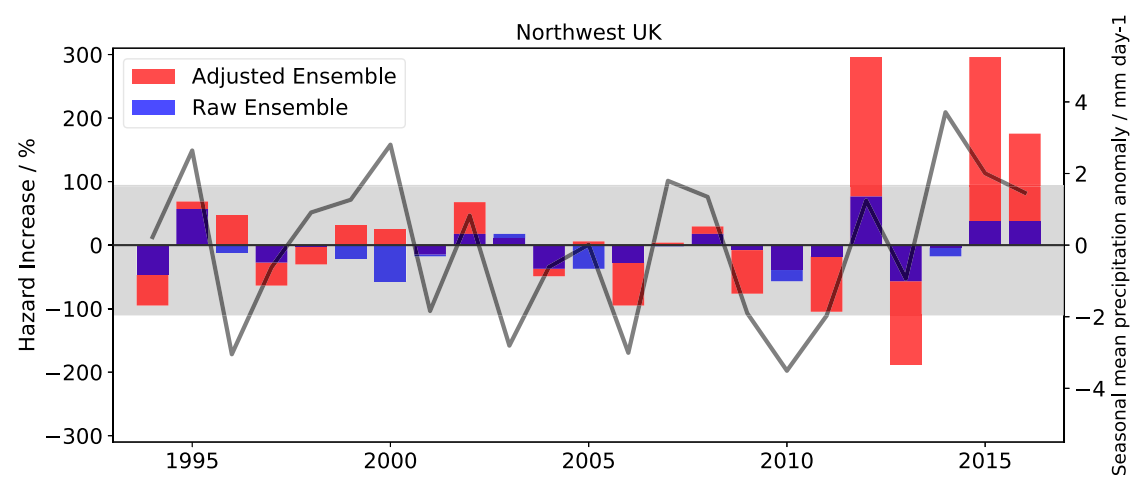

FIG. 12. Hazard increase (colored bars; left axis) as predicted by the raw and adjusted forecast systems for the northwestern United Kingdom over the hindcast period. Also shown is the coincident observed DJF seasonal mean precipitation over the northwestern United Kingdom (gray line; right axis). The gray shading indicates the middle tercile of the observed seasonal mean precipitation anomaly over the hindcast period (right axis).

hazard (i.e., a positive anomaly where the risk of a wet season in amplified and vice versa). There are a few years $(1996,2007$, and 2014) for which the predicted hazard increase is small while the observations show relatively large deviations from climatology. These correspond to years for which the observed NAO lies in the tail of the predicted ensemble. In these cases, the precipitation forecast ensemble is less likely to capture the observations. This highlights our reliance on the skill of the ensemble mean NAO forecast. The winter of 2009/10 had a record negative NAO in observations (Fereday et al. 2012), and although it was one of the most negative ensemble mean predictions in the hindcast period the observed value still lay in the tail of the ensemble distribution. It is seen that this season only had a relatively small increase in the risk of dry conditions.

If a particular region meets the conditions of having a significant correlation between the predicted ensemble mean and observed seasonal mean precipitation (Fig. 9) and an RPC of around 1 (Fig. 8), then these forecasts will provide improved estimates of the change in likelihood of different seasonal mean precipitation outcomes. The method developed here can provide many plausible realizations of daily sequences that are consistent with the predicted NAO, which allows the risk of extreme events as well as the expected seasonal mean precipitation to be assessed. The ultimate test of the forecasts will be to use them to initialize hydrological models and assess the skill of the hydrological forecasts over the hindcast period. Examples of where we are able to provide skillful seasonal precipitation forecasts are the United Kingdom, Ireland, Spain, Norway, and Sweden; however, this information will be most useful where the hydrological system is sensitive to the recent precipitation levels, rather than just the initial hydrological state.
For this assessment a hydrological model is needed. This future work will allow us to judge whether seasonal precipitation predictions are sufficiently improved to be useful for hydrological predictions.

\section{Conclusions}

In this paper we have described a method to improve the forecast of seasonal mean winter precipitation by increasing the magnitude of the predicted NAO signal. This adjusts for the known signal-to-noise problem present in several seasonal forecast models over the North Atlantic region (Scaife and Smith 2018) and means that we can now treat the individual ensemble members as plausible realizations (Siegert et al. 2016). For DJF we increased the size of the signal in the NAO predictions by a factor of 2.7, which results in an RPC of 0.99 . We use the large-scale seasonal mean circulation pattern (NAO) as the predictor of precipitation. This means that for skillful precipitation forecasts the observed seasonal mean precipitation needs to be well correlated with the NAO and the NAO needs to be well predicted by the forecast system. This restricts the use of this method to the winter season and certain geographical regions (United Kingdom, Ireland, Spain, Norway, and Sweden). We construct a large ensemble of daily precipitation realizations by using the adjusted NAO predictions to select analog months from the observational record. We show that when there is a large predicted NAO signal, the adjusted precipitation forecast distribution is further from the climatological distribution than the raw forecasts, and this increases the predicted likelihood of exceeding tercile thresholds. The forecasts perform best when there is a strong signal in the NAO predictions, which occurs in the observations. Although we have shown results here for DJF, we have 
also successfully applied the technique to the JanuaryMarch season. The ability to create a large ensemble of daily sequences of precipitation that are consistent with the skillful seasonal mean circulation forecasts is important for subsequent hydrological modeling and the assessment of the risk of extreme subseasonal events. Future work will be to assess hydrological forecasts over the hindcast period for specific regions and over basins of interest.

Acknowledgments. This research is part of IMPREX, a research project supported by the European Commission under the Horizon 2020 Framework Programme, with Grant 641811 (https://www.imprex.eu). We acknowledge the E-OBS dataset from the EU-FP6 project UERRA (http://www.uerra.eu) and the Copernicus Climate Change Service, and the data providers in the ECA\&D project (https://www.ecad.eu).

\section{REFERENCES}

Allan, R., and T. Ansell, 2006: A new globally complete monthly historical gridded mean sea level pressure dataset (HadSLP2): 1850-2004. J. Climate, 19, 5816-5842, https://doi.org/10.1175/ JCLI3937.1.

Anghileri, D., N. Voisin, A. Castelletti, F. Pianosi, B. Nijssen, and D. P. Lettenmaier, 2016: Value of long-term streamflow forecasts to reservoir operations for water supply in snowdominated river catchments. Water Resour. Res., 52, 42094225, https://doi.org/10.1002/2015WR017864.

Baker, L., L. Shaffrey, and A. A. Scaife, 2018a: Improved seasonal prediction of UK regional precipitation using atmospheric circulation. Int. J. Climatol., 38, e437-e453, https://doi.org/ 10.1002/joc.5382.

,,-- R. Sutton, A. Weisheimer, and A. Scaife, 2018b: An intercomparison of skill and overconfidence/underconfidence of the wintertime North Atlantic Oscillation in multimodel seasonal forecasts. Geophys. Res. Lett., 45, 7808-7817, https:// doi.org/10.1029/2018GL078838.

Beckers, J. V., A. H. Weerts, E. Tijdeman, and E. Welles, 2016: ENSO-conditioned weather resampling method for seasonal ensemble streamflow prediction. Hydrol. Earth Syst. Sci., 20, 3277-3287, https://doi.org/10.5194/hess-20-3277-2016.

Clark, R. T., P. E. Bett, H. E. Thornton, and A. A. Scaife, 2017: Skilful seasonal predictions for the European energy industry. Environ. Res. Lett., 12, 024002, https://doi.org/10.1088/17489326/AA57AB.

Copernicus, 2019: Climate Data Store. Copernicus, accessed 23 April 2019, https://cds.climate.copernicus.eu/cdsapp\#!/ dataset/seasonal-original-single-levels? tab = overview.

Day, G. N., 1985: Extended streamflow forecasting using NWSRFS. J. Water Resour. Plann. Manage., 111, 157-170, https://doi.org/ 10.1061/(ASCE)0733-9496(1985)111:2(157).

Dunstone, N., D. Smith, A. Scaife, L. Hermanson, R. Eade, N. Robinson, M. Andrews, and J. Knight, 2016: Skilful predictions of the winter North Atlantic Oscillation one year ahead. Nat. Geosci., 9, 809-814, https://doi.org/10.1038/ngeo2824.

Eade, R., D. Smith, A. Scaife, E. Wallace, N. Dunstone, L. Hermanson, and N. Robinson, 2014: Do seasonal-to-decadal climate predictions underestimate the predictability of the real world? Geophys. Res. Lett., 41, 5620-5628, https://doi.org/ 10.1002/2014GL061146.

ECA\&D, 2019: E-OBS Gridded Dataset. European Climate Assessment and Dataset, accessed 23 April 2019, https:// www.ecad.eu/download/ensembles/download.php.

Fereday, D., A. Maidens, A. Arribas, A. Scaife, and J. Knight, 2012: Seasonal forecasts of Northern Hemisphere winter 2009/10. Environ. Res. Lett., 7, 034031, https://doi.org/10.1088/ 1748-9326/7/3/034031.

Hamlet, A. F., D. Huppert, and D. P. Lettenmaier, 2002: Economic value of long-lead streamflow forecasts for Columbia River hydropower. J. Water Resour. Plann. Manage., 128, 91-101, https://doi.org/10.1061/(ASCE)0733-9496(2002)128:2(91).

Haylock, M. R., N. Hofstra, A. M. G. K. Tank, E. J. Klok, P. D. Jones, and M. New, 2008: A European daily high-resolution gridded data set of surface temperature and precipitation for 1950-2006. J. Geophys. Res., 113, D20119, https://doi.org/ 10.1029/2008JD010201.

Hurrell, J. W., 1995: Decadal trends in the North Atlantic Oscillation: Regional temperatures and precipitation. Science, 269, 676-679, https://doi.org/10.1126/science.269.5224.676.

— , Y. Kushnir, G. Ottersen, and M. Visbeck, 2003: An overview of the North Atlantic oscillation. The North Atlantic Oscillation: Climatic Significance and Environmental Impact, Vol. 134, Wiley, 1-35.

Karpechko, A. Y., K. A. Peterson, A. A. Scaife, J. Vainio, and H. Gregow, 2015: Skilful seasonal predictions of Baltic Sea ice cover. Environ. Res. Lett., 10, 044007, https://doi.org/10.1088/ 1748-9326/10/4/044007.

Li, H., L. Luo, E. F. Wood, and J. Schaake, 2009: The role of initial conditions and forcing uncertainties in seasonal hydrologic forecasting. J. Geophys. Res., 114, D04114, https://doi.org/ 10.1029/2008JD010969.

MacLachlan, C., and Coauthors, 2015: Global Seasonal Forecast System version 5 (GloSea5): A high-resolution seasonal forecast system. Quart. J. Roy. Meteor. Soc., 141, 1072-1084, https://doi.org/10.1002/qj.2396.

Marsh, T., C. Kirby, K. Muchan, L. Barker, E. Henderson, and J. Hannaford, 2016: The winter floods of 2015/2016 in the UK-A review. NERC/Centre for Ecology \& Hydrology Rep., 37 pp., http://nora.nerc.ac.uk/id/eprint/515303/1/N515303CR.pdf.

McCarthy, M., S. Spillane, S. Walsh, and M. Kendon, 2016: The meteorology of the exceptional winter of 2015/2016 across the UK and Ireland. Weather, 71, 305-313, https://doi.org/10.1002/ wea. 2823 .

Met Office, 2016: UK Storm Centre. Met Office, accessed 5 November 2018, https://www.metoffice.gov.uk/weather/ warnings-and-advice/uk-storm-centre/storm-desmond.

, 2019: Hadley Centre Sea Level Pressure dataset (HadSLP2). Met Office Hadley Centre observations datasets, accessed 23 April 2019, https://www.metoffice.gov.uk/hadobs/hadslp2/.

Palin, E. J., A. A. Scaife, E. Wallace, E. C. Pope, A. Arribas, and A. Brookshaw, 2016: Skillful seasonal forecasts of winter disruption to the UK transport system. J. Appl. Meteor. Climatol., 55, 325-344, https://doi.org/10.1175/JAMC-D-15-0102.1.

Scaife, A. A., and D. Smith, 2018: A signal-to-noise paradox in climate science. npj Climate Atmos. Sci., 1, 28, https://doi.org/ 10.1038/S41612-018-0038-4.

_, C. K. Folland, L. V. Alexander, A. Moberg, and J. R. Knight, 2008: European climate extremes and the North Atlantic Oscillation. J. Climate, 21, 72-83, https://doi.org/ 10.1175/2007JCLI1631.1. 
and Coauthors, 2014: Skillful long-range prediction of European and North American winters. Geophys. Res. Lett., 41, 2514-2519, https://doi.org/10.1002/2014GL059637.

—_, and Coauthors, 2017: Predictability of European winter 2015/2016. Atmos. Sci. Lett., 18, 38-44, https://doi.org/10.1002/asl.721.

Siegert, S., D. B. Stephenson, P. G. Sansom, A. A. Scaife, R. Eade, and A. Arribas, 2016: A Bayesian framework for verification and recalibration of ensemble forecasts: How uncertain is NAO predictability? J. Climate, 29, 995-1012, https://doi.org/ 10.1175/JCLI-D-15-0196.1.

Svensson, C., and Coauthors, 2015: Long-range forecasts of UK winter hydrology. Environ. Res. Lett., 10, 064006, https:// doi.org/10.1088/1748-9326/10/6/064006.

Thompson, V., N. J. Dunstone, A. A. Scaife, D. M. Smith, J. M. Slingo, S. Brown, and S. E. Belcher, 2017: High risk of unprecedented UK rainfall in the current climate. Nat. Commun., 8, 107, https://doi.org/10.1038/s41467-017-00275-3.

Thornton, H. E., A. A. Scaife, B. J. Hoskins, D. J. Brayshaw, D. M. Smith, N. Dunstone, N. Stringer, and P. E. Bett, 2019: Skilful seasonal prediction of winter gas demand. Environ. Res. Lett., 14, 024009, https://doi.org/10.1088/1748-9326/AAF338.
Viel, C., A.-L. Beaulant, J.-M. Soubeyroux, and J.-P. Céron, 2016: How seasonal forecast could help a decision maker: An example of climate service for water resource management. Adv. Sci. Res., 13, 51-55, https://doi.org/10.5194/ asr-13-51-2016.

Wood, A. W., and D. P. Lettenmaier, 2008: An ensemble approach for attribution of hydrologic prediction uncertainty. Geophys. Res. Lett., 35, L14401, https://doi.org/10.1029/2008GL034648.

Yiou, P., R. Vautard, P. Naveau, and C. Cassou, 2007: Inconsistency between atmospheric dynamics and temperatures during the exceptional 2006/2007 fall/winter and recent warming in Europe. Geophys. Res. Lett., 34, L21808, https://doi.org/10.1029/ 2007GL031981.

Yuan, X., E. F. Wood, and Z. Ma, 2015: A review on climatemodel-based seasonal hydrologic forecasting: Physical understanding and system development. Wiley Interdiscip. Rev.: Water, 2, 523-536, https://doi.org/10.1002/wat2.1088.

Zorita, E., and H. Von Storch, 1999: The analog method as a simple statistical downscaling technique: Comparison with more complicated methods. J. Climate, 12, 2474-2489, https://doi.org/10.1175/ 1520-0442(1999)012<2474:TAMAAS > 2.0.CO;2. 